\title{
Error due to atmospheric turbulence effects on solar diameter measurements performed with an astrolabe
}

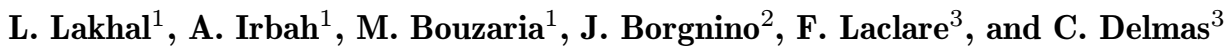 \\ 1 C.R.A.A.G, Observatoire d'Alger, BP. 63, Bouzaréah Alger, Algeria \\ e-mail: irbah@unice.fr \\ 2 Université de Nice-Sophia Antipolis, UMR 6525 Astrophysique, Parc Valrose, F-06108 Nice Cedex 2, France \\ 3 Observatoire de la Côte d'Azur, Département C.E.R.G.A, Avenue Copernic, F-06130 Grasse, France
}

Received March 15; accepted May 26, 1999

\begin{abstract}
Diameter measurements performed with the solar astrolabe are affected by instrumental and atmospheric effects. The problem is to know how these effects contribute to the error on diameter measurements. Thus, a numerical simulation is developed to bring some responses to this problem. For this purpose, synthetic images of the Sun similar to the ones obtained by the instrument through the Earth atmosphere are simulated. A fractal model is used to generate randomly perturbed wavefronts and therefore optical response of the whole system, atmosphere and instrument. Many sequences of solar images are then simulated for various observation conditions (Fried's parameter $r_{0}$, spatial coherence outer scale $L_{0}$ and atmospheric characteristic times). They are used to define the time of contact of the direct and reflected solar images which is fundamental in the solar astrolabe experiment. It is then studied as a function of the observation conditions relatively to the experimental characteristics (exposure time).
\end{abstract}

Key words: Sun: fundamental parameters - atmospheric effects - methods: numerical

\section{Introduction}

Many authors reported and discussed variations observed in the Sun's diameter measurements performed during the last three centuries (Toulmonde 1997). These results added greater interests to the solar diameter measurements and long term variations. Thus, many programs were born for solar geometry goals. Visual and photoelectric measurements have been made using instruments dedicated to the solar diameter measurements (Brown et al.

Send offprint requests to: J. Borgnino
1982; Bode et al. 1995; Laclare 1983; Laclare et al. 1980, 1996; Neckel 1995; Wittmann 1997; Wittmann et al. 1981, 1991). The mean values of solar radius reported from these programs were dispersed. The difference lied between a few tenths to more than two arcseconds. Moreover, variations have not always been noted from the observation series of the solar programs. Using the observations recorded with the solar diameter monitor (Brown et al. 1982) during the period 1982 to 1987, Brown \& Christensen-Dalsgaard (1998) did not observe any variations. In their work, they used special methods in order to eliminate some atmospheric effects. However, in opposite, variations were observed in diameter measurements performed with the solar astrolabe (Delache et al. 1985; Laclare et al. 1996).

The astrolabe experiment of Calern Observatory (France) has begun in 1975, when the first measurements were obtained. Since the beginning of the solar program up to now, visual observations were made regularly while the CCD ones began in 1988. Figure 1 represents the visual solar data recorded at Calern Observatory astrolabe during the period 1975 to 1996 . It is clearly shown an oscillation with a period of about ten years corresponding probably to the solar cycle but in opposite phase. Others new solar astrolabe programs have been then developed from these new exciting results (Laclare et al. 1996; Leister \& Benevides Soares 1990; Noël 1995; Sanchez et al. 1995). Several studies were also developed in order to analyze (Moussaoui et al. 1998; Delache et al. 1985; Gavryusev et al. 1994) and to valide the recorded data (Laclare et al. 1996; Irbah et al. 1994). For this purpose and to improve the astrolabe experiment, all effects which are able to cause variations or degradations of the solar data, are required to be studied.

The present work is a study of the instrumental and atmospheric turbulence effects to the contribution to diameter measurement errors when observing with a solar astrolabe. It is developed using a numerical 


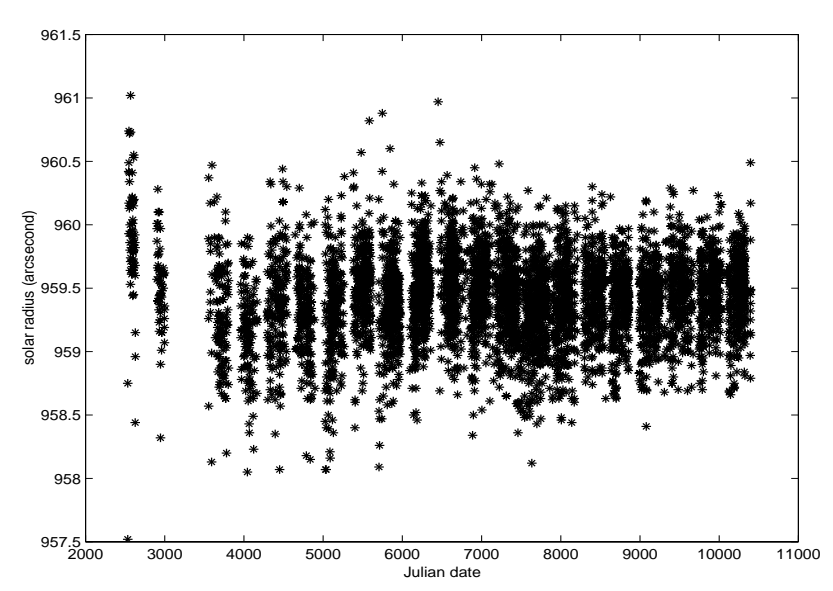

Fig. 1. Solar radius measurements obtained using the Calern Observatory astrolabe during the period 1975 to 1996

simulation where the principal atmospheric parameters are introduced. The effect of each parameter is studied separately in order to evaluate its proper contribution to the global diameter error. Section 2 presents the simulation of solar images as observed at the astrolabe through the atmospheric turbulence. In the simulation, a fractal model is used to generate randomly perturbed wavefronts and therefore point spread functions ( $p s f$ ) of the whole atmosphere-instrument. In Sect. 3, we present the steps leading to the instant of the Sun transit by the small circle needed for the diameter measurement with the astrolabe. Finally, we expose and discuss the results obtained for the contribution of each atmospheric parameter to the error on the diameter estimation.

\section{Synthetic images as observed at the solar astrolabe}

In this section, solar images with similar characteristics as those recorded at the astrolabe through the atmospheric turbulence are simulated. The optical response of the whole system atmosphere and instrument is then calculated using a fractal model to generate the randomly perturbed wavefronts. It is used to build solar images in various seeing conditions and with different exposure times. Let us first present the synthetic image of the Sun which is needed for the simulation.

\subsection{Synthetic image of the Sun}

Several models of the solar limb darkening function exist. The model we use to build the synthetic image of the Sun is given by (Allen 1973):

$O_{\lambda}(\alpha)=O_{\lambda}(0)\left[1-u_{2}-v_{2}+u_{2} \cos (\alpha)+v_{2} \cos ^{2}(\alpha)\right]$

where $O_{\lambda}(\alpha)$ is the intensity of the solar continuum at an angle $\alpha$ from the centre of the disk. $\alpha$ represents the
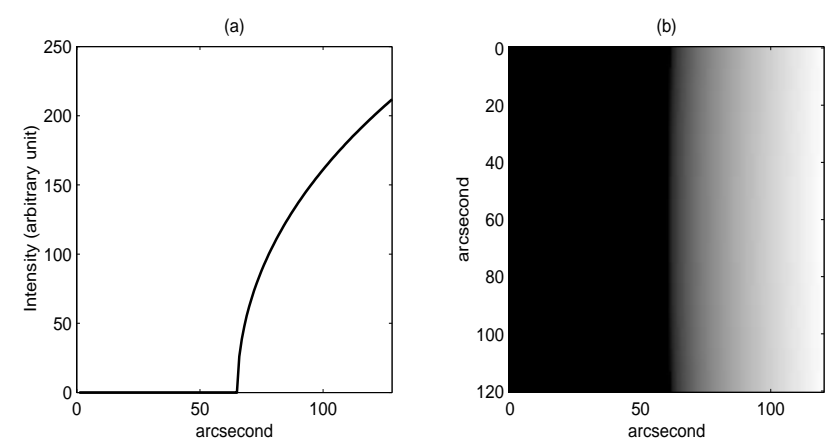

Fig. 2. Solar limb darkening function a) and the synthetic image of the sun $\mathbf{b}$ )

angle between a Sun's radius vector and the line of sight. $\alpha=0$ corresponds to the centre of the disk and $\alpha=\frac{\pi}{2}$ to the solar edge. The ratio $\frac{O_{\lambda}(\alpha)}{O_{\lambda}(0)}$, which varies with the wavelength $\lambda$ defines the limb darkening. For the used observation wavelength $(\lambda=0.55 \mu \mathrm{m})$, the constants $u_{2}$ and $v_{2}$ are respectively equal to 0.93 and -0.23 . The plot in Fig. 2a shows the solar limb darkening function where the intensity was normalized between 0 and 255 for our needed calculations. The Fig. $2 \mathrm{~b}$ represents a solar image sample where the field size is equal to 128 by $128^{\prime \prime 2}$.

\subsection{Simulation of the optical response of the whole system, atmosphere and instrument using a fractal model}

The $p s f$ of the whole system instrument and atmosphere is needed to generate the solar image sequences as recorded through the atmosphere in various observation conditions. We present first, the fractal model used to generate the two-dimensional randomly perturbed wavefronts and then the deduced optical responses.

2.2.1. The randomly perturbed wavefront synthesis using a fractal model

The turbulence follows a fractal structure. The model used to generate the randomly perturbed wavefront function $\varphi$ is based on a mid point displacement algorithm (Lane et al. 1992; Beaumont et al. 1996). To simulate a $L$ by $L$ phase screen, the first step is to choose three points $\phi_{1}$, $\phi_{2}$ and $\phi_{3}$ disposed at the summits of an isoceles triangle with base and height equal to $2 L$ (Fig. 3). Phase function values at these points are Gaussian random variables with zero means and with variances given by:

$\sigma^{2}\left(\phi_{1}\right)=D_{\phi}(\sqrt{5} L)-\frac{1}{2} D_{\phi}(2 L)$

and

$\sigma^{2}\left(\phi_{2}\right)=\sigma^{2}\left(\phi_{3}\right)=\frac{1}{2} D_{\phi}(2 L)$ 


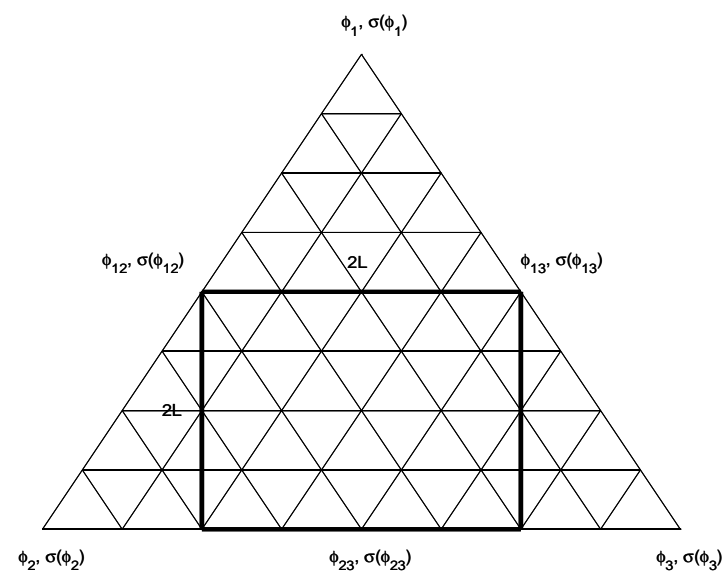

Fig. 3. The fractal interpolation grid used to generate the perturbed wavefronts

where $D_{\phi}(r)=\left\langle|\varphi(x)-\varphi(x+r)|^{2}\right\rangle=6.88\left(\frac{r}{r_{0}}\right)^{\frac{5}{3}} *$

$\left[1-1.485\left(\frac{r}{L_{0}}\right)^{\frac{1}{3}}+5.383\left(\frac{r}{L_{0}}\right)^{2}-6.281\left(\frac{r}{L_{0}}\right)^{\frac{7}{3}}\right]$

$<>$ denotes a mean average. $D_{\phi}(r)$ is the phase structure function deduced from the Kolmogorov's law and the Von Karman model (Ziad 1993). $r_{0}$ is the Fried's parameter which qualifies the observations (seeing) (Fried 1966) and $L_{0}$ the spatial coherence outer scale of the wavefronts (Borgnino 1990).

Equations (2), (3) and (4) show that $\phi_{1}, \phi_{2}$ and $\phi_{3}$ follow the theoretical phase structure function deduced from the Kolmogorov turbulence law since:

$$
\begin{aligned}
& \left\langle\left|\phi_{1}-\phi_{2}\right|^{2}\right\rangle=\left\langle|\varphi(x)-\varphi(x+\sqrt{5} L)|^{2}\right\rangle=D_{\phi}(\sqrt{5} L) \\
& \left\langle\left|\phi_{3}-\phi_{1}\right|^{2}\right\rangle=\left\langle|\varphi(x)-\varphi(x+\sqrt{5} L)|^{2}\right\rangle=D_{\phi}(\sqrt{5} L) \\
& \left\langle\left|\phi_{3}-\phi_{2}\right|^{2}\right\rangle=\left\langle|\varphi(x)-\varphi(x+2 L)|^{2}\right\rangle=D_{\phi}(2 L) .
\end{aligned}
$$

Three new points $\phi_{12}, \phi_{13}$ and $\phi_{23}$ of the phase screen are generated midway between each existing points by a process of linear interpolation and addition of independent random variables $\varepsilon_{1}, \varepsilon_{2}$ and $\varepsilon_{3}$, such as:

$$
\begin{aligned}
\phi_{12} & =\frac{1}{2}\left(\phi_{1}+\phi_{2}\right)+\varepsilon_{1} \\
\phi_{13} & =\frac{1}{2}\left(\phi_{1}+\phi_{3}\right)+\varepsilon_{2} \\
\phi_{23} & =\frac{1}{2}\left(\phi_{2}+\phi_{3}\right)+\varepsilon_{3}
\end{aligned}
$$

$$
\sigma^{2}\left(\varepsilon_{1}\right)=\sigma^{2}\left(\varepsilon_{2}\right)=D_{\phi}\left(\frac{\sqrt{5}}{2} L\right)-\beta D_{\phi}(\sqrt{5} L)
$$

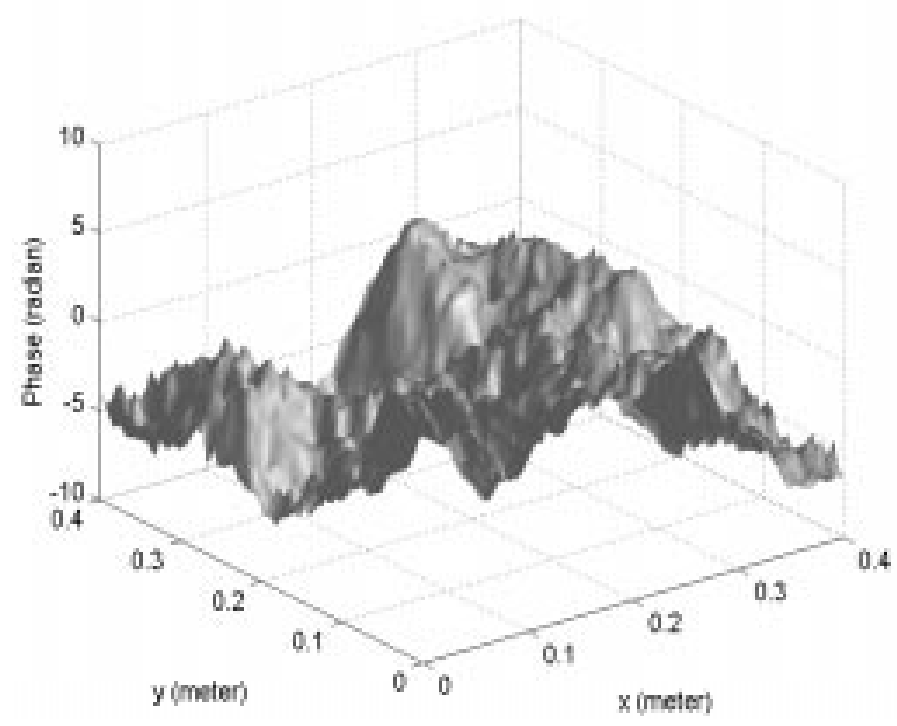

Fig. 4. Perturbed wavefront sample generated for $r_{0}=4 \mathrm{~cm}$ and $L_{0}$ infinite

and

$\sigma^{2}\left(\varepsilon_{3}\right)=D_{\phi}(L)-\beta D_{\phi}(2 L)$

where $\beta$ is a semi-empirical coefficient equal to 0.25 .

The iterative procedure interpolation/displacement is then repeated in each new generated triangle until the desired numbers of samples are obtained. The needed $L$ by $L$ phase screen is extracted from the middle of the base of the original isoceles triangle (Fig. 3). The Fig. 4 shows the simulation of a perturbed wavefront sample using the model. It is simulated for a Fried's parameter $r_{0}$ equal to $4 \mathrm{~cm}$ and a spatial coherence outer scale supposed infinite.

\subsubsection{The simulated point spread function}

The psf $S$ of the whole system telescope and atmosphere is given by the square modulus of the Fourier Transform $(F T)$ of the complex amplitude $\Psi$ limited to the pupil area:

$S(\vec{\theta})=\left[F T_{\text {pupil }}(\Psi(\vec{\xi}))\right]^{2}$

where

$\Psi(\vec{\xi})=\exp (i \varphi(\vec{\xi}))$.

Equation (8) assumed the near field approximation (Roddier 1981). $\varphi$ is the turbulent phase generated with the fractal model, $\vec{\xi}$ is the angular frequency vector considered in the pupil plane and $\vec{\theta}$ the angular coordinate vector in the focal plane.

$S(\vec{\theta})$ corresponds to an image of speckles. In Fig. 5 are represented four typical short exposure speckle images obtained using the procedure developed in the previous section. They are simulated for Fried's parameters $r_{0}$ equal 
(a)

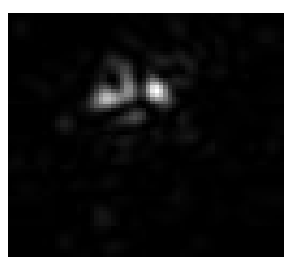

(b)

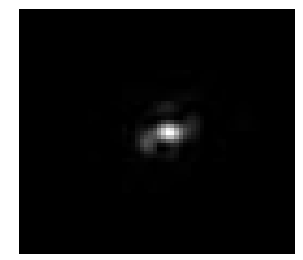

(c)

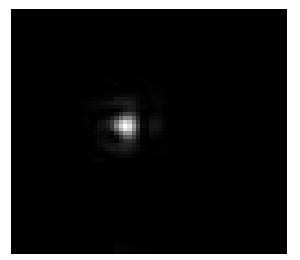

(d)

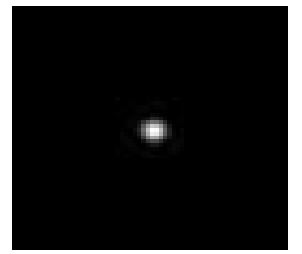

Fig. 5. Speckle images obtained from the fractal model. The image field is equal to 64 by $64^{\prime \prime 2}$ and the Fried's parameter equal respectively to $2 \mathbf{a}), 4 \mathbf{b}), 6 \mathbf{c}$ ) and $10 \mathrm{~cm} \mathrm{~d}$ )

(a)

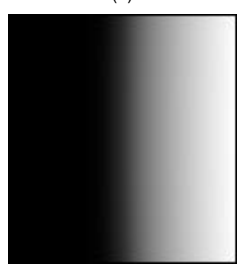

(b)

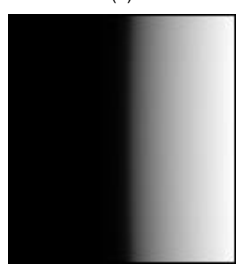

(c)

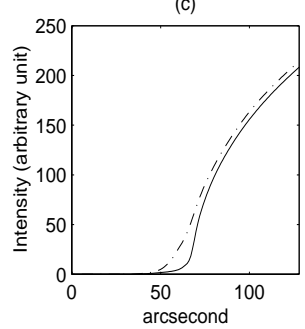

(a)

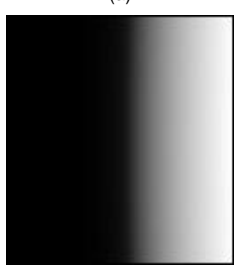

(b)

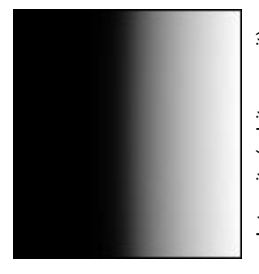

(c)

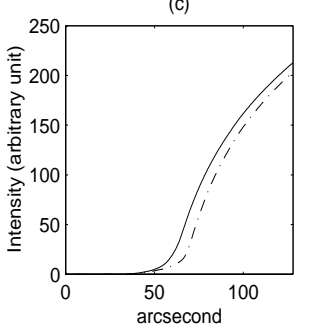

Fig. 6. Simulated solar images recorded with a short exposure time and $r_{0}$ equal to $3 \mathbf{a}$ ) and $7 \mathrm{~cm} \mathrm{~b}$ ). Solar limbs extracted from images recorded in these seeing conditions (dashed line: $r_{0}=3 \mathrm{~cm}$ and solid line: $\left.r_{0}=7 \mathrm{~cm}\right) \mathbf{c}$ )

respectively to 2, 4, 6 and $10 \mathrm{~cm}$ and with a pupil aperture $D$ equal to $10 \mathrm{~cm}$. The spatial coherence outer scale of the used randomly perturbed wavefronts has an infinite value. Large boiling and displacements of the speckle centroids are observed for small values of $r_{0}$ while for $r_{0}$ equal to the pupil aperture, the speckle image is similar to the diffraction pattern of the instrument.

We are then able to simulate psf's of the whole system telescope and atmosphere in various seeing conditions and also for different integration times (average of short exposure samples).

\subsection{Simulation of recorded solar images in various observation conditions}

Solar images as recorded through the terrestrial atmosphere are simulated. Assuming isoplanatism, a solar image is obtained by the convolution of the synthetic image of the Sun (see Sect. 2.1) with the psf of the imaging system (atmosphere and telescope):

$I(\vec{\theta})=O(\vec{\theta}) \otimes S(\vec{\theta})$ with $|\vec{\theta}| \leq \theta_{0}$

where $\otimes$ is the convolution symbol and $\theta_{0}$ the isoplanatic angle.

We use this formalism to build solar images recorded in various seeing conditions and with different CCD integration times. The spatial coherence outer scale of the generated perturbed wavefronts is infinite. The pupil aperture $D$ and the observation wavelength are respectively taken equal to $10 \mathrm{~cm}$ and $0.55 \mu \mathrm{m}$. These kind of images will be useful in the next section to develop the error study on solar diameter measurement. They have similar properties

Fig. 7. Simulated solar image recorded in same seeing conditions $\left(r_{0}=5 \mathrm{~cm}\right)$ but with different exposure times $(t \leq \tau$ a) and $t \gg \tau \mathbf{b})$ ). Solar limbs extracted from images recorded with exposure time respectively lower (solid line) and higher (dashed line) than the atmospheric characteristic time $\tau$ c)

as those recorded with the solar astrolabe (Laclare et al. 1996; Laclare \& Merlin 1991).

2.3.1. Solar images recorded in different seeing conditions but with a short exposure time

In this case, solar images are simulated using various Fried's parameters $r_{0}$ (Fig. 6) but integration times short enough to freeze the turbulent motion of the atmosphere i.e. the integration time $t$ is less than the characteristic evolution time of the atmosphere $\tau$ (Aime et al. 1986). For a given simulated image sequence, Fried's parameter $r_{0}$ remains constant but images are obtained with independent $p s f$ 's.

2.3.2. Solar images recorded in same seeing conditions but with different exposure times

Long exposure solar images are obtained by the average over a great number of short exposure frames. The integration time $t$ of the long exposure images is then equal to $n \tau$ where $n$ is the number of averaged images and $\tau$ the atmospheric characteristic time. Figure 7 shows the simulated solar images recorded in same seeing conditions $\left(r_{0}=5 \mathrm{~cm}\right)$ but with different integration times.

\section{Error on the diameter measurements performed with} a solar astrolabe. Results and discussion

We use in this section, the previous developments to study the error behavior of solar diameter measurements performed with a solar astrolabe in various seeing conditions 

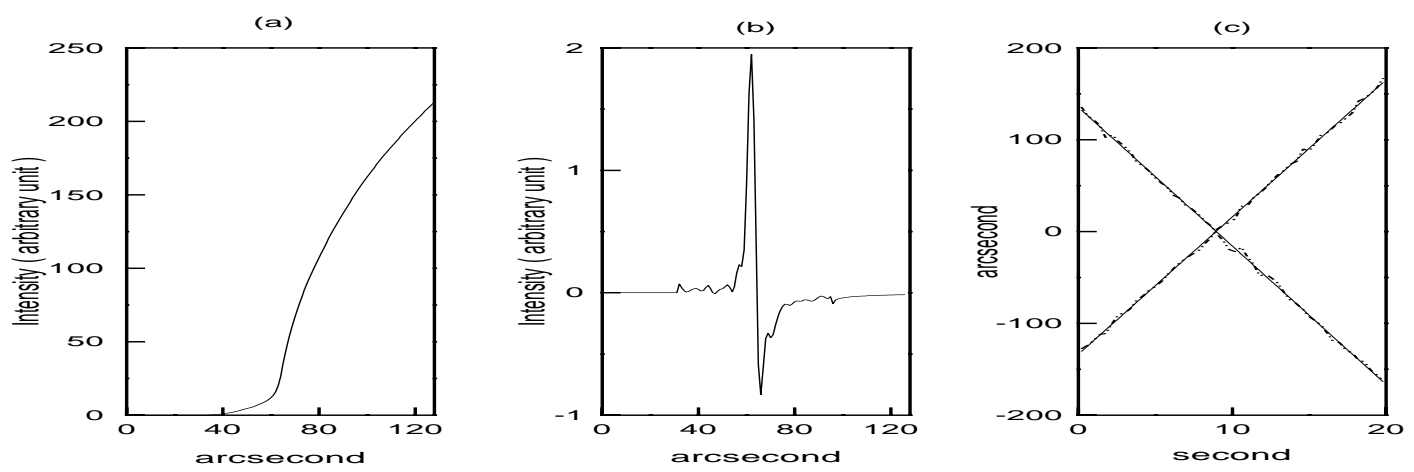

Fig. 8. Analysis of an image sequence composed of short exposure frames recorded for a Fried's parameter equal to $3 \mathrm{~cm}$. a) Typical simulated solar limb extracted from a solar image. b) Second derivative of the solar limb function and definition of the inflexion point. c) Reconstructed trajectories of both direct and reflected solar images approximated by straight lines using least-square fits

and for different integration times of the CCD camera. It is useful to recall first the basic properties of the solar astrolabe experiment.

\subsection{The solar astrolabe experiment}

The diameter measurement principle consists in the determination of the time transits of the solar edges by the small circle localized at an altitude $Z$ defined by the angle of the astrolabe prism (Laclare 1983). The solar diameter is deduced from the time interval between the two solar limb transits by the same small circle. Near the transit of the Sun by the small circle, the astrolabe gives two images of the object: a direct and a reflected ones. Each of them moves in the astrolabe focal plane due to the diurnal motion. The instant when the solar edge crosses the small circle corresponds to the contact of the two images. In the solar experiment, each solar image is formed on a CCD camera using a rotating shutter (Laclare \& Merlin 1991). The solar image is analyzed by the acquisition system which extracts in real time, the solar edge. The edge is determined by the inflexion points of the solar limb function given by each image line.

For one solar image, the recorded data represent the solar edge associated to the corresponding time acquisition. For each solar edge transit by the small circle, data consist in a sequence of about 100 images recorded during about 20 seconds. The solar trajectory is determined from the data for both direct and reflected images. The intersection point of the trajectories gives the time when the solar edge crosses the small circle.

\subsection{Simulation of solar data recorded in various observation conditions}

To simulate observations at the astrolabe, solar image sequences recorded in various observation conditions are needed. For given observation conditions, a simulated sequence is constituted with 50 direct images and 50 reflected ones. Each simulated solar image has a size of 128 by $128^{\prime \prime 2}$. The pupil aperture $D$ and the observation wavelength are also respectively equal to $10 \mathrm{~cm}$ and $0.55 \mu \mathrm{m}$. A Sun mean velocity of $15^{\prime \prime}$ by second of time is taken in order to introduce the diurnal motion. To each solar image is associated a recorded time taking in reference the first simulated one. The acquisition sampling is fixed to $20 \mathrm{sec}-$ onds. Each solar image of the sequence is then analyzed and the solar edge extracted. As in the solar experiment, the edge is defined as the zero of the second derivative of each solar limb function assimilated to an image line. The edge associated to the corresponding time allows to build the trajectories for both direct and reflected solar images. The trajectories are after that approximated by straight lines using least-square fits and the desired intersection point so determined (Fig. 8).

\subsection{Results and discussion}

Since the diameter measurement is deduced from the two instants of the solar edge transit by the small circle, we will develop our error study around the time transit behavior. Using the previous developments, many sequences of solar images have been simulated in various observation conditions (Fried's parameter $r_{0}$, outer scale $L_{0}$, exposure time $t$ ) (see Sect. 2.3). The sequence analysis leads to the transit time of the Sun by the small circle directly linked to the observation conditions. Thus, because of temporal fluctuations observed on the trajectories, the time transit is not well defined (Irbah et al. 1994). We define the error $\Delta t$ associated to the transit time $t_{0}$ as:

$\Delta t=\left|t_{0}-t_{\text {true }}\right|$

where $t_{\text {true }}$ denotes the true contact time of solar images obtained in ideal seeing conditions. 
(a)

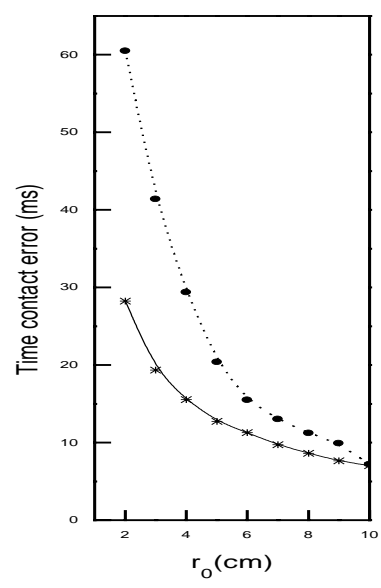

(b)

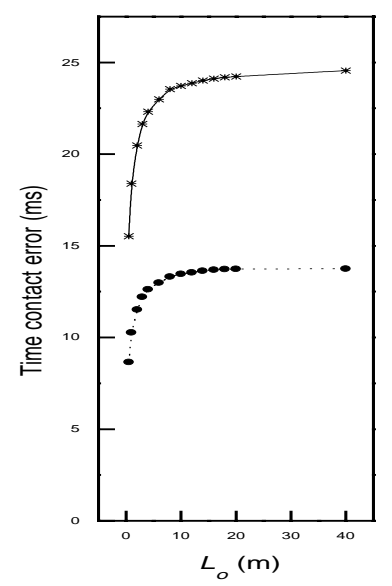

(c)

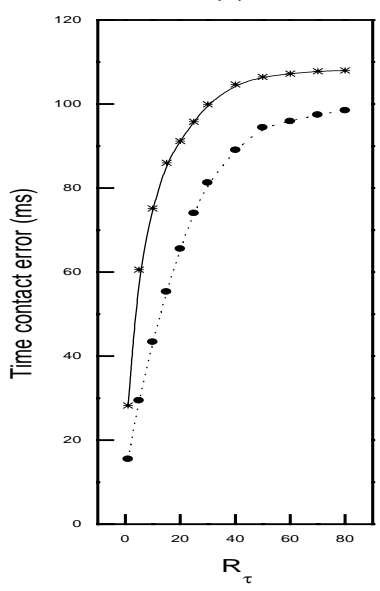

Fig. 9. Time contact error with: a) the seeing (-*: short and .•.: long exposure frames), b) the outer scale $L_{0}\left(-^{*}: r_{0}=2 \mathrm{~cm}\right.$ and $\left.. \bullet: r_{0}=4 \mathrm{~cm}\right)$ and $\left.\mathbf{c}\right)$ the parameter $R_{\tau}\left(-^{*}: r_{0}=2 \mathrm{~cm}\right.$ and $\left.. \bullet .: r_{0}=4 \mathrm{~cm}\right)$ (see text)

We will now analyze the error behavior with the observation conditions.

\subsubsection{Error due to the seeing}

Solar image sequences composed of short and long exposure frames are used to study the error behavior with the seeing. They are simulated for various seeing conditions and supposing an infinite outer scale of the randomly perturbed wavefronts. For a given Fried's parameter $r_{0}$, the error $\Delta t$ is determined over an average of 50 transit times deduced from each kind of the simulated solar sequences. The plot of the Fig. 9a shows the error behavior with the seeing as obtained from the simulation. In each case, a decrease of the error with good seeing conditions is observed. The use of long exposure frames in the experiment gives however, a greater error on the time contact than when using short exposure frames. This is better observed on the figure for poor seeing conditions. Indeed, in case of short exposure frames, the error is greater than $28 \mathrm{~ms}$ when the $r_{0}$ value is about $2 \mathrm{~cm}$ and falls down to about $13 \mathrm{~ms}$ for $r_{0}$ equal to $5 \mathrm{~cm}$ (see Table 1). The precision improvement is then equal to $54 \%$. The error still decreases to about $9 \mathrm{~ms}$ for $r_{0}$ equal to $8 \mathrm{~cm}$ and improves the precision of about $68 \%$. When using long exposure frames, the error which is equal to $61 \mathrm{~ms}$ when $r_{0}$ is equal to $2 \mathrm{~cm}$, falls down to about $20 \mathrm{~ms}$ for $r_{0}$ equal to $5 \mathrm{~cm}$. The precision improvement is then equal to $67 \%$. The error decreases to about $11 \mathrm{~ms}$ for $r_{0}$ equal to $8 \mathrm{~cm}$ which gives a precision improvement of about $82 \%$.

For mean seeing conditions at Calern Observatory $\left(r_{0}=4 \mathrm{~cm}\right)$ (Irbah et al. 1994), the error is about $16 \mathrm{~ms}$ if short exposure frames are used and about $29 \mathrm{~ms}$ in case of long exposure frames. According to the considered Sun mean velocity (see Sect. 3.2), the error on the solar diameter measurement is respectively equal to $0.5^{\prime \prime}$ and $0.9^{\prime \prime}$ when short and long exposure frames are used (see Table 2).

3.3.2. Error due to the spatial coherence outer scale of the perturbed wavefronts

To study this effect, image sequences composed of short exposure frames recorded in same seeing conditions are generated for different spatial coherence outer scales. The error $\Delta t$ is determined over an average of 30 transit time samples. The Fig. 9b represents the time contact error behavior with the outer scale $L_{0}$ in two different seeing conditions $\left(r_{0}=2\right.$ and $\left.4 \mathrm{~cm}\right)$. In each case, the error increases with the outer scale $L_{0}$ in order to reach a limit value. It is also observed from the figure that this parameter has a significant effect only when it has small values $\left(L_{0} \leq 8 \mathrm{~m}\right)$. It is limited after that by the error induced by the seeing. For the mean seeing conditions of the Calern Observatory, the error on the time transit is respectively equal to about 12 and $13 \mathrm{~ms}$ for $L_{0}$ equal to 2 and $8 \mathrm{~m}$ (see Table 1). It remains then constant to about $14 \mathrm{~ms}$ when $L_{0}$ values are greater than $20 \mathrm{~m}$. This corresponds to an error on the solar diameter measurement of about $0.1^{\prime \prime}$ when $L_{0}$ takes values from 2 to $20 \mathrm{~m}$ (see Table 2).

3.3.3. Error due to the exposure time relatively to the observation conditions

A similar study has been also developed using sequences simulated in case of different exposure times relatively to the turbulence characteristic time. The outer scale is supposed infinite and two seeing conditions are considered $\left(r_{0}=2\right.$ and $\left.4 \mathrm{~cm}\right)$. We define the parameter $R_{\tau}$ as the ratio between the exposure time and the characteristic time. The Fig. $9 \mathrm{c}$ shows the time difference $\Delta t$ behavior with the 
Table 1. Error on the time contact

\begin{tabular}{|c|c|c|c|c|c|c|c|c|}
\hline$r_{0}(\mathrm{~cm})$ & \multicolumn{2}{|c|}{$\begin{array}{c}\Delta t(\mathrm{~ms}) \\
\left(L_{0} \text { infinite }\right)\end{array}$} & $L_{0}(\mathrm{~m})$ & \multicolumn{2}{c|}{$\begin{array}{c}\Delta t(\mathrm{~ms}) \\
\left(R_{\tau} \leq 1\right)\end{array}$} & & \multicolumn{2}{c|}{$\begin{array}{c}\Delta t(\mathrm{~ms}) \\
\left(L_{0} \text { infinite }\right)\end{array}$} \\
\hline \hline & $R_{\tau} \leq 1$ & $R_{\tau} \succ 1$ & & $r_{0}=2 \mathrm{~cm}$ & $r_{0}=4 \mathrm{~cm}$ & & $r_{0}=2 \mathrm{~cm}$ & $r_{0}=4 \mathrm{~cm}$ \\
\cline { 2 - 3 } \cline { 5 - 8 } 2 & 28.2 & 60.5 & 0.5 & 15.5 & 8.7 & 1 & 28.2 & 15.5 \\
3 & 19.3 & 41.4 & 1 & 18.4 & 10.3 & 5 & 60.6 & 29.4 \\
4 & 15.5 & 29.4 & 2 & 20.5 & 11.5 & 10 & 75.1 & 43.4 \\
5 & 12.7 & 20.4 & 3 & 21.6 & 12.2 & 15 & 86.0 & 55.3 \\
6 & 11.3 & 15.5 & 4 & 22.3 & 12.6 & 20 & 91.2 & 65.6 \\
7 & 9.7 & 13.0 & 6 & 23.0 & 13.0 & 25 & 95.8 & 74.1 \\
8 & 8.6 & 11.2 & 8 & 23.6 & 13.3 & 30 & 100.0 & 81.2 \\
9 & 7.6 & 9.9 & 10 & 23.7 & 13.5 & 40 & 104.7 & 89.1 \\
10 & 7.0 & 7.3 & 12 & 23.9 & 13.5 & 50 & 106.5 & 94.4 \\
& & & 14 & 24.0 & 13.6 & 60 & 107.2 & 95.9 \\
& & & 16 & 24.1 & 13.7 & 70 & 107.8 & 97.4 \\
& & & 40 & 24.6 & 13.7 & 80 & 108.0 & 98.5 \\
\hline
\end{tabular}

Table 2. Error on the solar diameter measurements

\begin{tabular}{|c|c|c|c|c|c|c|c|c|}
\hline$r_{0}(\mathrm{~cm})$ & \multicolumn{2}{|c|}{$\begin{array}{c}\Delta D\left({ }^{\prime \prime}\right) \\
\left(L_{0} \text { infinite }\right)\end{array}$} & $L_{0}(\mathrm{~m})$ & \multicolumn{2}{|c|}{$\begin{array}{c}\Delta D\left(^{\prime \prime}\right) \\
\left(R_{\tau} \leq 1\right)\end{array}$} & $R_{\tau}$ & \multicolumn{2}{|c|}{$\begin{array}{c}\Delta D\left({ }^{\prime \prime}\right) \\
\left(L_{0} \text { infinite }\right)\end{array}$} \\
\hline & $\overline{R_{\tau} \leq 1}$ & $\overline{R_{\tau} \succ 1}$ & & $r_{0}=2 \mathrm{~cm}$ & $r_{0}=4 \mathrm{~cm}$ & & $r_{0}=2 \mathrm{~cm}$ & $r_{0}=4 \mathrm{~cm}$ \\
\hline 2 & 0.9 & 1.8 & 0.5 & 0.5 & 0.3 & 1 & 0.9 & 0.5 \\
\hline 3 & 0.6 & 1.2 & 1 & 0.6 & 0.3 & 5 & 1.8 & 0.9 \\
\hline 4 & 0.5 & 0.9 & 2 & 0.6 & 0.4 & 10 & 2.3 & 1.3 \\
\hline 5 & 0.4 & 0.6 & 3 & 0.7 & 0.4 & 15 & 2.6 & 1.7 \\
\hline 6 & 0.3 & 0.5 & 4 & 0.7 & 0.4 & 20 & 2.7 & 2.0 \\
\hline 7 & 0.3 & 0.4 & 6 & 0.7 & 0.4 & 25 & 2.9 & 2.2 \\
\hline 8 & 0.3 & 0.3 & 8 & 0.7 & 0.4 & 30 & 3.0 & 2.4 \\
\hline 9 & 0.2 & 0.3 & 10 & 0.7 & 0.4 & 40 & 3.1 & 2.7 \\
\hline 10 & 0.2 & 0.2 & 12 & 0.7 & 0.4 & 50 & 3.2 & 2.8 \\
\hline & & & 14 & 0.7 & 0.4 & 60 & 3.2 & 2.9 \\
\hline & & & 16 & 0.7 & 0.4 & 70 & 3.2 & 2.9 \\
\hline & & & 40 & 0.7 & 0.4 & 80 & 3.2 & 3.0 \\
\hline
\end{tabular}

$R_{\tau}$ parameter. For the considered seeing conditions, it is observed a growth of the time difference $\Delta t$ for small $R_{\tau}$ values which reaches a limit value. For the mean seeing condition of Calern Observatory, the error on the time transit is equal to $15.5 \mathrm{~ms}\left(0.5^{\prime \prime}\right.$ on the diameter measurements) for $R_{\tau}$ equal to 1 (short exposure frames) which corresponds to the error due to the seeing $\left(r_{0}=4 \mathrm{~cm}\right)$. It is multiplied by a factor 2.8 then 4.2 when $R_{\tau}$ value goes from 10 to 20 . It remains to a constant value of about $100 \mathrm{~ms}\left(3^{\prime \prime}\right.$ on the diameter measurements) for the higher values of the $R_{\tau}$ parameter. Consequently, the precision loss is about $280 \%$ when $R_{\tau}$ goes from 1 to 10 and about $420 \%$ when it becomes equal to 20 (see Tables 1 and 2).

We can notice finally that, although long exposure frames are obtained in best seeing conditions, the precision is worse than that we obtain in case of poor seeing conditions but with short exposure frames (with $R_{\tau} \leq 1$ and $r_{0}=2 \mathrm{~cm}, \Delta t=28 \mathrm{~ms}$ while with $R_{\tau}=10$ and $\left.r_{0}=4 \mathrm{~cm}, \Delta t=43 \mathrm{~ms}\right)$. Thus, to have at least same accuracy's time contact than for poor seeing conditions, $R_{\tau}$ needs to be less than a factor 5 . Thus, the $R_{\tau}$ parameter appears to be fundamental in the solar astrolabe experiment. A knowledge of time turbulence properties is then suitable to adjust experimental parameters and improve accuracy of diameter measurement performed with the solar astrolabe. Table 1 summarizes the results on the time contact error obtained from this simulation while Table 2 presents the same results but for the solar diameter measurements.

\section{Conclusion}

Solar diameter measurements performed at Calern Observatory astrolabe during two solar cycles (22 years) shows variations. These variations leaded to many studies and one cause often advanced is the Earth atmosphere. It is then in order to quantify how the atmospheric turbulence effects contribute to the error in the solar diameter estimations that the present work was developed. It is based around a numerical simulation of the measurements at the solar astrolabe in various observation conditions. The first step was to simulate images of the Sun as those observed at the solar astrolabe through the Earth atmosphere. A synthetic image of 
the Sun was then needed. It was built using a model of the limb darkening function. A fractal model was after used to generate wavefronts randomly perturbed by the atmospheric turbulence and therefore optical responses of the whole system atmosphere and instrument. They were generated for various observation conditions and used to obtain the solar images. The second step was to simulate the astrolabe experiment and to define the needed parameters for the diameter measurements. The observed trajectories of both direct and reflected images are then built for various observation conditions. These are defined by the Fried's parameter, the spatial coherence outer scale of wavefronts and the ratio of the exposure time to the atmospheric characteristic time. The intersection point of the trajectories corresponding to the Sun transit time by the small circle, was then determined linked to the observation conditions. The study of the error due to atmospheric turbulence effects was developed around the transit time behavior with the observation conditions. Results show a decrease of the error with good seeing conditions whatever the used exposure times. The precision is however better in case of short exposure times than for long ones. A growth of the error is observed with the values of spatial coherence outer scale. It is significant only for small values of the outer scale and limited after by the error induced by the seeing. The error behavior is more critical with the exposure time relatively to the turbulence characteristic time. The error increases rapidly with the time ratio in order to reach a limit value. Thus, to have at least the same accuracy on the diameter measurement in poor seeing conditions that what we have with good seeing but using long exposure frames, this parameter needs to be less than a factor 5 . This ratio seems then to be fundamental for the precision of the measurements with the solar astrolabe. To improve measurement diameter accuracy with the solar astrolabe, knowledge of time turbulence properties is suitable to adjust experimental parameters.

Acknowledgements. This work has been performed with support of the Observatoire d'Alger (C.R.A.A.G) and the French
Foreign affair Ministry in the framework of scientific cooperation between France and Algeria (contract 96 MDU 357).

\section{References}

Aime C., Borgnino J., Kadiri S., et al., 1986, J. Opt. Soc. Am. A 3, 1001

Allen C.W, Astrophys. Quant., 1973, Athlone (ed.), p. 169

Beaumont H., Aime C., Sanchez L., Lanteri H., 1996, ESO Workshop on Science with the VLT interferometer. Garching-bei München

Bode H.J., Buchner R., Mushardt M., 1995, Sterne Weltraum 34,646

Borgnino J., 1990, Appl. Opt. 29, 1863

Brown T.M., Elmore D.F., Lacey L., Hull H., 1982, Appl. Opt. 21, 19, 3588

Brown, Dalsgaard, 1998, ApJL v 500, L195

Delache Ph., Laclare F., Sadsaoud H., 1985, Nat 317, 548

Fried D.L., 1966, J. Opt. Soc. Am. 56, 1372

Gavryusev V., Gavryuseva E., Delache P., Laclare F., 1994, A\&A 286, 305

Irbah A., Laclare F., Borgnino J., Merlin G., 1994, Solar Phys. 149, 213

Laclare F., Demarcq J., Chollet F., 1980, C. R. Acad. Sci. Paris, 291B, 189

Laclare F., 1983, A\&A 125, 200

Laclare F., Merlin G, 1991, C. R. Acad. Sci. Paris, 313 SII, 323

Laclare F., Delmas C., Coin J.P., Irbah A., 1996, Solar Phys. 166,211

Lane R.G, Glindemann A., Dainty J.C., 1992, Waves Random Media 2, 209

Leister N.V., Benevides Soares P., 1990, C. R. Acad. Sci. Paris, 313 SII, 399

Moussaoui R., Irbah A., Abdelatif T., et al., 1998 (submitted to Solar Phys.)

Neckel H., 1995, Solar Phys. 156, 7

Noël F., 1995, A\&AS 113, 131

Roddier F., 1981, Progr. Opt. XIX, Wolf (ed.), No. 18, 283

Sanchez M., Parra F., Soler M., Soto R., 1995, A\&AS 110, 351

Toulmonde M., 1997, A\&A 325, 1174

Wittmann A., Bonet Navarro J.A., Wöhl H., 1981, The physics of Sunspots, Sacramento Peak Nat Observ., p. 424

Wittmann A., Alge E., Bianda M., 1991, Solar Phys. 135, 243

Wittmann A., 1997, Solar Phys. 171, 231

Ziad A., 1993, Univ. de Nice-Sophia Antipolis, Thesis 